\title{
THE ALL-SKY EXTRAGALACTIC X-RAY FOREGROUND
}

\author{
ELIHU BOLDT \\ NASA/Goddard Space Flight Center, Greenbelt, MD 20771
}

\section{Introduction}

Observations of galaxies in the IR and optical (Lynden-Bell et al. 1989) suggest that the $600 \mathrm{~km} / \mathrm{s}$ peculiar velocity of the LG (Local Group of galaxies) arises mainly from a foreground of anisotropically distributed mass within $z=0.013$ (i.e., $\mathrm{HR}<4000 \mathrm{~km} / \mathrm{s})$. Since the X-ray luminosity of bright extragalactic X-ray sources provides a good mass measure of the radiating objects involved and can be observed relatively free of galactic obscuration effects, such sources are likely candidates for serving as reliable tracers of the total underlying mass (i.e., dark as well as visible) responsible for the acceleration of the LG. In this connection, we note that the local gravitational dipole implied by the fifty X-ray brightest clusters of galaxies at $z>0.013$ considered by Lahav et al. (1989) is relatively small compared with that inferred from the only three clusters at lower redshifts. Since the local space density of AGN (Active Galactic Nuclei) is about two orders of magnitude greater than rich clusters, however, such compact sources have the potential of providing a vastly improved statistical sample for tracing mass in the low-redshift region of particular interest. Furthermore, recent dipole analysis of the X-ray flux from bright AGN observed with HEAO-1 A2 indicates that they are indeed strong tracers of this matter (Miyaji and Boldt 1990). The implications of this for the very pronounced large-scale foreground anisotropies to be measured via low-redshift AGN resolved in more sensitive all-sky surveys are explored.

For the total extragalactic X-ray sky, i.e. including the relatively large Cosmic $\mathrm{X}$-ray Background (CXB) as well as the contribution of resolved sources, the dipole moment is small compared to the monopole. Because of the large peculiar velocity of the LG, however, it is found that the currently estimated value for this total dipole moment can already be used to set remarkably severe constraints on the volume emissivity arising from all X-ray sources within the present epoch. This provides significant limits on the CXB contributions of all source populations that are well represented in the local universe.

\section{Dipole Moments}

We know that the extragalactic X-ray sky is dominated by a nearly isotropic, unresolved CXB and suspect that there exists a proper frame in which it shows precise global isotropy. If this frame is anchored to that of the microwave background, then the solar velocity $v_{0}=380 \mathrm{~km} / \mathrm{s}$ relative to it yields an apparent dipole anisotropy (Compton and Getting 1935) in the observed surface brightness (B), with an apex

Y. Kondo (ed.), Observatories in Earth Orbit and Beyond, 451-455.

(C) 1990 Kluwer Academic Publishers. Printed in The Netherlands. 
having galactic coordinates $l=267 \mathrm{deg}, b=+50 \mathrm{deg}$ (Lynden-Bell et al. 1988). The dipole moment (D) of this distribution over the whole sky is defined as

$$
D \equiv \int_{4 \pi} B \cos (\theta) \mathrm{d} \Omega
$$

where $\theta$ is the angle relative to the preferred axis. The monopole $(M)$ is:

$$
M=\int_{4 \pi} B \mathrm{~d} \Omega
$$

For a power-law energy spectrum of index (a) the dipole/monopole ratio $(D / M)$ is

$$
D / M=\left(v_{0} / c\right)[1+(\alpha / 3)]
$$

For $\alpha=0.4$, the value appropriate to the $2-10 \mathrm{keV}$ CXB, $D / M=1.4 \times 10^{-3}$ (i.e., a small number). Although this is compatible with the $\mathrm{CXB}$ global anisotropy estimated with HEAO-1 A2 (Shafer 1983), the precision of that determination is strongly limited by foreground fluctuations due to unresolved sources and possible extended regions of weakly enhanced surface brightness (Jahoda and Mushotzky 1989).

In sharp contrast to the small value of $D / M$ for the unresolved $\mathrm{CXB}$ as a whole, the $\mathrm{D} / \mathrm{M}$ ratio derived from the all-sky extragalactic foreground of discrete sources (at $\mathrm{RH}<4000 \mathrm{~km} / \mathrm{s}$ ) is very large. For examining this foreground we define

$$
D \equiv \sum_{i} S_{i} \cos \left(\theta_{i}\right)
$$

and

$$
M \equiv \sum_{i} S_{i}
$$

where $S_{i}$ is the flux from each individual source and $\theta_{i}$ the angle with respect to a direction $(l=268 \mathrm{deg}, b=+27 \mathrm{deg})$ defined by the peculiar velocity of the $\mathrm{LG}$ (Lahav et al 1989). In particular, for galaxies (mainly spirals) observed with IRAS, $D / M \sim 0.2$ (Lahav et al. 1988). For optically observed galaxies (ellipticals as well as spirals), $D / M \sim 0.4$ (Lynden-Bell et al. 1989). With sources observed in X-rays $D / M \sim 0.5$ for AGN's (Miyaji and Boldt 1990) and $D / M \gtrsim 0.5$ for the statistically weaker sample of clusters (Lahav et al. 1989).

According to linear perturbation theory (Peebles 1980), the peculiar velocity $\mathbf{v}$ of the $\mathbf{L G}$ is given by

$$
\mathbf{v} \sim \mathrm{g}\left[2 /(3 H)\left(\rho / \rho_{c}\right)^{-0.4}\right.
$$

where $H$ is the Hubble constant, $\rho$ is the mean total mass density, $\rho_{c}$ is the critical closure density and $\mathrm{g}$ is the net gravitational acceleration vector at the $\mathrm{LG}$. The magnitude of $\mathrm{g}$ is given by

$$
|g|=\sum_{i} g_{i} \cos \left(\theta_{i}\right)=4 \pi G(\rho / b)(D / M) R
$$


where the sum indicated is over gravity contributions $\left(g_{i}\right)$ arising from all mass elements within a radial distance $(R), G$ is the gravitational constant and $b=$ (luminous contrast) $/$ (mass contrast) is a "bias" factor characterizing radiative tracers of mass.

In particular, using the values of $D / M$ observed in various electromagnetic bands we infer from equations $(6)$ and $(7)$ that $b$ (X-ray) $\geq b$ (optical) $\approx 2 \times[b($ IR $)]$, where $b$ (optical) $\approx 2.7\left(\rho / \rho_{c}\right)^{0.6}$; see Lynden-Bell et al. $(1989)$.

\section{Local X-ray Volume Emissivity}

Although the dipole fit to the global anisotropy of the CXB is compatible with what is expected from the Compton-Getting effect (Shafer 1983), well within statistical errors, the actual "best fit" apex $(l=313 \mathrm{deg}, b=+38 \mathrm{deg})$ is closer to that of the peculiar velocity of the LG than it is to the direction of our own (solar system) motion relative to the proper frame of the microwave background. This suggests that some finite portion of this dipole moment arises from the anisotropic distribution of unresolved X-ray sources which trace the underlying matter responsible for the peculiar velocity of the LG. In any event, the dipole moment resulting from anisotropically distributed unresolved X-ray sources can not exceed that for the overall X-ray sky. As such, the dipole moment of the overall X-ray sky can be used to set a limit on the local volume emissivity corresponding to the complete population of present-epoch discrete X-ray sources (including those as yet unresolved) as well as all diffuse emission. In particular, since X-ray emission appears to be a strong tracer of matter, we can use the all-sky dipole moment $\left(D_{\text {All }}\right)$ and the observed peculiar velocity $(v)$ of the LG to set a significant limit on the local X-ray volume emissivity $(q \equiv M / R$ ). To do this we note from equations $(6)-(7)$ that

$$
q<(H / v)\left(D_{\mathrm{All}}\right) /\left[b\left(\rho_{c} / \rho\right)^{0.6}\right]
$$

where $D_{\text {All }}$ is the magnitude of the total dipole moment obtained from the vector addition of the dipole moments associated with the Compton-Getting effect (CG), Resolved $\underline{A} G N(R A)$ and the apparent surface brightness enhancement identified with the Great $\underline{A}$ tractor (GA), viz:

$$
\mathbf{D}_{\mathrm{All}}=\mathbf{D}_{\mathrm{CG}}+\mathbf{D}_{\mathrm{RA}}+\mathbf{D}_{\mathrm{GA}} \text {. }
$$

The $4 \%$ enhancement in X-ray surface brightness over a 40 deg diameter region centered at a position ( $l=310 \mathrm{deg}, b=+10 \mathrm{deg}$ ) identified with the GA (Jahoda and Mushotzky 1989) corresponds to a dipole moment in that direction of magnitude

$$
D_{\mathrm{GA}}=1.2 \times 10^{-3} M_{\mathrm{CXB}},
$$

where $M_{\mathrm{CXB}}=6.6 \times 10^{-7} \mathrm{erg} \mathrm{cm}^{-2} \mathrm{~s}^{-1}$ is the CXB monopole $(2-10 \mathrm{keV})$. (Such direct effects are discussed in detail by Goicoechea and Martin-Mirones (1990).) For the Compton-Getting effect we obtain via equation (3) that

$$
M_{\mathrm{CG}}=1.4 \times 10^{-3} M_{\mathrm{CXB}} .
$$


The dipole moment obtained from resolved AGN (Miyaji and Boldt 1990) is in a direction ( $l=313 \mathrm{deg}, b=+38 \mathrm{deg}$ ) $39 \mathrm{deg}$ away from the velocity vector characterizing the peculiar motion of the $L G$ and has a magnitude

$$
D_{\mathrm{RA}}(2-10 \mathrm{keV})=5 \times 10^{-10} \mathrm{erg} \mathrm{cm}^{-2} \mathrm{~s}^{-1}
$$

Using equations (9)-(12) we obtain that $D_{\text {All }}$ is in a direction ( $l=297 \mathrm{deg}$, $b=+35 \mathrm{deg}$ ) somewhat closer to that of the peculiar velocity of the LG and has a magnitude

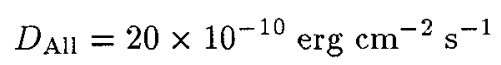

From equations (8) and (13) we obtain an upper limit to the local volume X-ray emissivity arising from all sources, viz:

$$
q<5.9 \times 10^{38} \mathrm{~h} \mathrm{erg} \mathrm{s}^{-1} \mathrm{Mpc}^{-3} \text { for all sources }
$$

where $h=H /\left(50 \mathrm{~km} \mathrm{~s}^{-1} \mathrm{Mpc}^{-1}\right)$ and we have taken $b=2.7\left(\rho / \rho_{c}\right)^{0.6}$ for all sources of X-ray emission. It is interesting to compare this upper limit on the local composite volume X-ray emissivity with that coming from bright AGN having L(2-10 keV) $>10^{43} \mathrm{~h}^{-2} \mathrm{erg} \mathrm{s}^{-1}$ (Piccinotti et al.1982), viz :

$$
q=2 \times 10^{38} \mathrm{herg} \mathrm{s}^{-1} \mathrm{Mpc}^{-3} \text { for bright AGN. }
$$

[The local volume X-ray emissivity $(2-10 \mathrm{keV}$ ) arising from Abell clusters is $\leq$ $1 \times 10^{38} \mathrm{~h} \mathrm{erg} \mathrm{s}^{-1} \mathrm{Mpc}^{-3}$ (McKee 1980; Piccinotti et al. 1982).] The upper limit given by equation (14) provides a severe constraint on other possible populations of extragalactic X-ray sources in the present epoch, such as the moderate redshift faint radio galaxies proposed by Helfand et al. (1989) to be major CXB contributors. In particular, without evolution, all such other source populations can make no more than a $20 \%$ contribution to the CXB $(2-10 \mathrm{keV})$.

\section{Future Prospects}

Assuming a present-epoch density of about $10^{-6} \mathrm{~h}^{3} \mathrm{Mpc}^{-3}$ for rich $(R \geq 1)$ clusters (Bahcall 1988) the number expected within the critical foreground region $(H R<4000 \mathrm{~km} / \mathrm{s}$ ) corresponds to only a couple of objects or so. This is clearly too few for their effective utilization in tracing the anisotropic distribution of matter responsible for the peculiar motion of the $L G$. On the other hand, for an AGN space density of about $10^{-4} \mathrm{~h}^{3} \mathrm{Mpc}^{-3}$ for $L_{x}>10^{42} \mathrm{~h}^{-2}$ erg s${ }^{-1}$ (Persic et al. 1989 ) the number expected in this foreground region would be a few hundred. And the number expected over twice the critical depth (i.e., within $\mathrm{HR} \approx 8000 \mathrm{~km} \mathrm{~s}^{-1}$ ) would be more than a thousand, ample for studying the dipole growth with respect to redshift in the interesting region where preliminary results based on a limited sample of AGN (Miyaji and Boldt 1990) indicate the approach of a plateau (i.e., saturation). The spectral homogeneity (2-10keV) of AGN and the good correlation of their X-ray luminosity with the mass of the underlying supermassive blackhole 
(Wandel and Mushotzky 1986) renders these sources potentially powerful and interesting tracers of all gravitational mass. A suitable all-sky X-ray foreground survey of AGN could be used to answer the question: "How do supermassive blackholes trace the overall underlying mass distribution responsible for the peculiar velocity of the Local Group?"

The sensitivity (2-10 keV) required for the all-sky survey of foreground AGN

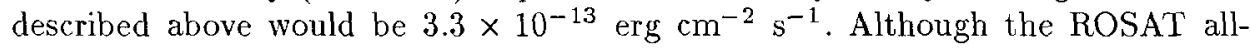
sky survey is expected to be at this sensitivity level below $2 \mathrm{keV}$, it does not have sufficient response within the higher energy band needed for an unbiased AGN survey (i.e., one minimizing the effects of galactic absorption and spectral variations). Deepening the all-sky survey to $H R \approx 30,000 \mathrm{~km} / \mathrm{s}$ (i.e., $z \approx 0.1$ ) would require a sensitivity of about $2 \times 10^{-14} \mathrm{erg} \mathrm{cm}^{-2} \mathrm{~s}^{-1}(2-10 \mathrm{keV})$, comparable to that of the high sensitivity small-field survey (1-3 keV) of the Einstein Observatory.

\section{Acknowledgements}

I thank Ofer Lahav and Takamitsu Miyaji for stimulating discussions.

\section{References}

Bahcall, N.: 1988, Ann. Rev. Astron. Astrophys. 26, 631

Compton, A. and Getting, I.: 1935, Phys. Rev. 47, 817

Goicoechea L., and Martin-Mirones, J.: 1990, MNRAS , in press

Helfand, D. et al.: 1989, Bull. AAS 121, 1220

Jahoda, K. and Mushotzky, M.: 1989, Astroph. J. 346, 638

Lahav, O., Rowan-Robinson, M. and Lynden-Bell, D.: 1988, MNRAS 234, 677

Lahav, O., Edge, A., Fabian, A. and Putney, A.: 1989, MNRAS 238, 881

Lynden-Bell, D. et al.: 1988, Astroph. J. 326, 19

Lynden-Bell, D., Lahav, O. and Burstein, D.: 1989, MNRAS 241, 325

McKee, J. et al.: 1980, Astroph. J. 242, 843

Miyaji, T. and Boldt, E.: 1990, Astroph. J.(Letters), in press

Peebles, P. J. E.: 1980, The Large Scale Structure of the Universe, Princeton University

Press, Priceton

Persic, M. et al.: 1989, Astroph. J. 344, 125

Shafer, R.: 1983, PhD thesis, University of Maryland, NASA TM 85029

Wandel, A. and Mushotzky, R.: 1986, Astroph. J. 306, L61 